\title{
Effect of Intermittent Hypercapnia on Respiratory Control in Rat Pups
}

\author{
Justin A. Steggerda Catherine A. Mayer Richard J. Martin \\ Christopher G. Wilson \\ Department of Pediatrics, Case Western Reserve University, Cleveland, Ohio, USA
}

\section{Key Words}

Hypoxia $\cdot$ Hypercapnia $\cdot$ Neonatal apnea $\cdot$ Chemoreceptors

\begin{abstract}
Preterm infants are subject to fluctuations in blood gas status associated with immature respiratory control. Intermittent hypoxia during early postnatal life has been shown to increase chemoreceptor sensitivity and destabilize the breathing pattern; however, intermittent hypercapnia remains poorly studied. Therefore, to test the hypothesis that intermittent hypercapnia results in altered respiratory control, we examined the effects of daily exposure to intermittent hypercapnia on the ventilatory response to subsequent hypercapnic and hypoxic exposure in neonatal rat pups. Exposure cycles consisted of 5 min of intermittent hypercapnia $\left(5 \% \mathrm{CO}_{2}, 21 \% \mathrm{O}_{2}\right.$, balance $\mathrm{N}_{2}$ ) followed by 10 min of normoxia. Rat pups were exposed to 18 exposure cycles each day for 1 week, from postnatal day 7 to 14 . We analyzed diaphragm electromyograms (EMGs) from pups exposed to subsequent acute hypercapnic $\left(5 \% \mathrm{CO}_{2}\right)$ and hypoxic $\left(12 \% \mathrm{O}_{2}\right)$ challenges. In response to a subsequent hypercapnia challenge, there was no significant difference in the ventilatory response between control and intermittent hypercapnia-exposed groups. In contrast, intermittent hypercapnia-exposed rat pups showed an enhanced ventilatory response to hypoxic challenge with an increase in minute EMG to $118 \pm$ $14 \%$ of baseline versus $107 \pm 13 \%$ for control pups $(p<0.05)$
\end{abstract}

We speculate that prior hypercapnic exposure may increase peripheral chemoreceptor response to subsequent hypoxic exposures and result in perturbed neonatal respiratory control.

Copyright $\odot 2009$ S. Karger AG, Basel

\section{Introduction}

Fluctuations in blood gas status are a common phenomenon in both spontaneously breathing and ventilated preterm infants. This is a result of immature respiratory control, compounded by ineffective ventilation associated with impaired pulmonary function [1]. As a consequence, preterm infants are typically exposed to episodes of hypercapnia and hypoxia, which are repetitive in nature. The aim of this study, therefore, was to elucidate the specific effects of recurrent hypercapnia on ventilatory responses to subsequent hypercapnic and hypoxic episodes in an immature animal model.

The ventilatory response to a single hypercapnic exposure has been extensively studied and is diminished in apneic preterm infants $[2,3]$ and during early postnatal life in both preterm infants and rat pups [4-6]. The characteristic increase in tidal volume is accompanied by a decrease in respiratory frequency during hypercapnic exposure in preterm infants $[7,8]$. Neonatal rats exhibit a similar response to acute hypercapnic exposure [9]; how-

\section{KARGER}

두 2009 S. Karger AG, Basel

Fax +41613061234 E-Mail karger@karger.ch www.karger.com www.karger.com/neo
Richard J. Martin, MD

Division of Neonatology, Department of Pediatrics

Rainbow Babies and Children's Hospital, Suite 3100

11100 Euclid Drive, Cleveland, OH 44106 (USA)

Tel. +1 216844 3387, Fax +1 216844 3380, E-Mail Rxm6@case.edu 
ever, the effect of repetitive hypercapnic exposures on respiratory control during early development is unclear.

In contrast to repetitive hypercapnia, the consequences of repetitive hypoxic exposure have been extensively studied. In rat pups, episodic exposure to hypoxia increases the magnitude of the respiratory response to a subsequent hypoxic challenge [10]. This is consistent with our observation and that of others that preterm infants with a greater number of apneic episodes exhibit an increased ventilatory response to hypoxic exposure $[11,12]$, and that enhanced peripheral chemoreceptor activity in response to hypoxic exposure may destabilize respiratory control [10]. While repetitive hypoxia undoubtedly modulates peripheral chemoreceptor function, repetitive hypercapnia may also alter respiratory neural output. In piglets, episodic exposure to combined hypercapnia-hypoxia has been shown to enhance the minute ventilation response to subsequent hypoxic challenges [13]. However, the contributions of repetitive hypercapnia exposure, alone, to changes in breathing pattern remain unclear. We therefore exposed rat pups to episodic hypercapnia in order to test the hypothesis that repetitive hypercapnia modulates the response to subsequent hypercapnic and hypoxic challenges, with the potential to destabilize respiratory neural output in early postnatal life.

\section{Methods}

We studied Sprague-Dawley rat pups aged postnatal days 7 to 15 (Harlan, Indianapolis, Ind.; Charles River, Wilmington, Mass., USA), which were kept with their mothers throughout the exposures. Each litter was housed in the Case Western Reserve University (CWRU) Animal Resource Center in a room with timecontrolled lighting of $12 \mathrm{~h}$ of light and $12 \mathrm{~h}$ of darkness. All animal protocols were approved by the CWRU Institutional Animal Care and Use Committee.

\section{Exposure Protocol}

In this study, rat pups in both control $(\mathrm{n}=10)$ and exposed ( $\mathrm{n}=14$ ) groups were taken from multiple litters ( 7 total). Cycles of hypercapnic exposure $\left(5 \% \mathrm{CO}_{2}, 21 \% \mathrm{O}_{2}\right.$, balance $\left.\mathrm{N}_{2}\right)$ were of 5 min duration, followed by $10 \mathrm{~min}$ of room air. Eighteen cycles of hypercapnic exposure occurred daily from postnatal day 7 to postnatal day 14 . The exposures were performed by placing the cage with mother and pups in a sealed Plexiglas ${ }^{\circledR}$ box connected to an air tank that provided the hypercapnic mixture. Exposure conditions were maintained using a flowmeter (Cole-Parmer, Vernon Hills, Ill., USA), with a maintained pressure between 40 and 50 psi. Control animals were kept with their mother and exposed to room air only from birth.

We verified that the exposure protocol induced hypercapnia in rat pups by exposing a subgroup of animals to our exposure protocol and then obtaining a blood gas sample at the end of hy- percapnia or from control animals. Using exposed $(n=9)$ and control pups $(\mathrm{n}=10)$, we found that the protocol resulted in a significantly elevated $\mathrm{PaCO}_{2}$ in exposed rat pups (57.3 \pm 6.9 Torr) compared to control pups $(46.0 \pm 3.6$ Torr; $\mathrm{p}<0.001)$.

\section{Acute Animal Preparation}

Both exposed and control groups underwent the same surgical preparation designed to measure physiologic responses at the conclusion of intermittent hypercapnic exposure on day 15. Animals were anesthetized with intraperitoneal urethane at $0.1 \mathrm{ml} /$ $10 \mathrm{~g}$ body weight (ethyl carbamate; $4 \mathrm{~g} / \mathrm{kg}$ i.p., Sigma, St. Louis, Mo., USA). Each animal's body temperature was regulated with a feedback controlled water blanket to maintain the animal's temperature at approximately $39^{\circ} \mathrm{C}$ (measured using a thermistor placed between the water blanket and the rat) throughout the procedure. An arterial catheter was placed in the carotid artery for blood gas assessment. A bilateral vagotomy was performed to remove the influence of stretch receptors on respiratory timing by entrainment to the ventilator. A tracheostomy was performed and the animal was mechanically ventilated with a volume ventilator at an average rate of 38 breaths/min and a volume of $0.5 \mathrm{~cm}^{3}$ / breath (Harvard Apparatus, Holliston, Mass., USA) based on previous work in our laboratory [14]. The animals were not paralyzed as we were using electromyography as our measure of inspiratory drive, and this rate matched the ventilator rate approximately 1 : 1. A fine-gauge, stainless steel, bipolar, hook electrode was inserted into the costal diaphragm for measurement of electromyographic activity. Electromyographic activity was digitally recorded using PowerLab hardware (ADI Instruments) and Chart software.

\section{Physiological Protocols}

After the surgical preparation was finished, the animal was allowed a recovery period before baseline measures were acquired to insure stable baseline breathing. After $5 \mathrm{~min}$ of baseline recording, each animal was exposed to the $5 \% \mathrm{CO}_{2}, 21 \% \mathrm{O}_{2}$, balance $\mathrm{N}_{2}$, hypercapnic mixture for $5 \mathrm{~min}$. All gas mixtures were administered via the inflow valve on the ventilator. The pup was then switched to room air for a 10 -min recovery period. Each animal was next exposed to the $12 \% \mathrm{O}_{2}$, balance $\mathrm{N}_{2}$, hypoxic mixture for $5 \mathrm{~min}$. This was followed by another $10 \mathrm{~min}$ of recovery. The sequence of the challenges was varied to avoid serial-dependency problems for repeated measures; 11 animals were subjected to hypercapnic challenge followed by the hypoxic challenge, while 9 were first exposed to the hypoxic and then hypercapnic gas mixtures. We then withdrew $0.2 \mathrm{ml}$ of arterial blood from the carotid catheter for blood gas analysis (ABL blood gas analyzer, Radiometer America, Westlake, Ohio, USA). Blood was analyzed to ensure $\mathrm{pH}$ levels were between 7.2 and 7.4 and the base excess was between 0 and $-10 \mathrm{mmol} / \mathrm{l}$ at the conclusion of the protocol. Four animals were excluded prior to data analysis, as these conditions were not met. This resulted in analysis of data from 8 control and 12 intermittent-hypercapnia-exposed rat pups. Each rat pup was euthanized by a lethal dose of $\mathrm{KCl}$, administered through the arterial catheter while under deep anesthesia.

Data Analysis

We used Matlab software to integrate chart data and analyze frequency and integrated electromyograms (EMGs) from 10 consecutive respiratory cycles prior to and following the administra- 
Fig. 1. a Visual representation of experimental protocol with aggregate durations. b Integrated EMG ( $\left.\int E M G\right)$ of a control rat pup during baseline, hypercapnic challenge, and hypoxic challenge, respectively. c Integrated EMG ( $\int \mathrm{EMG}$ ) of a hypercapnia-exposed rat pup during baseline, hypercapnic, and hypoxic challenges. Note the greater amplitude response to hypoxic challenge in the rat pup previously exposed to intermittent hypercapnia.

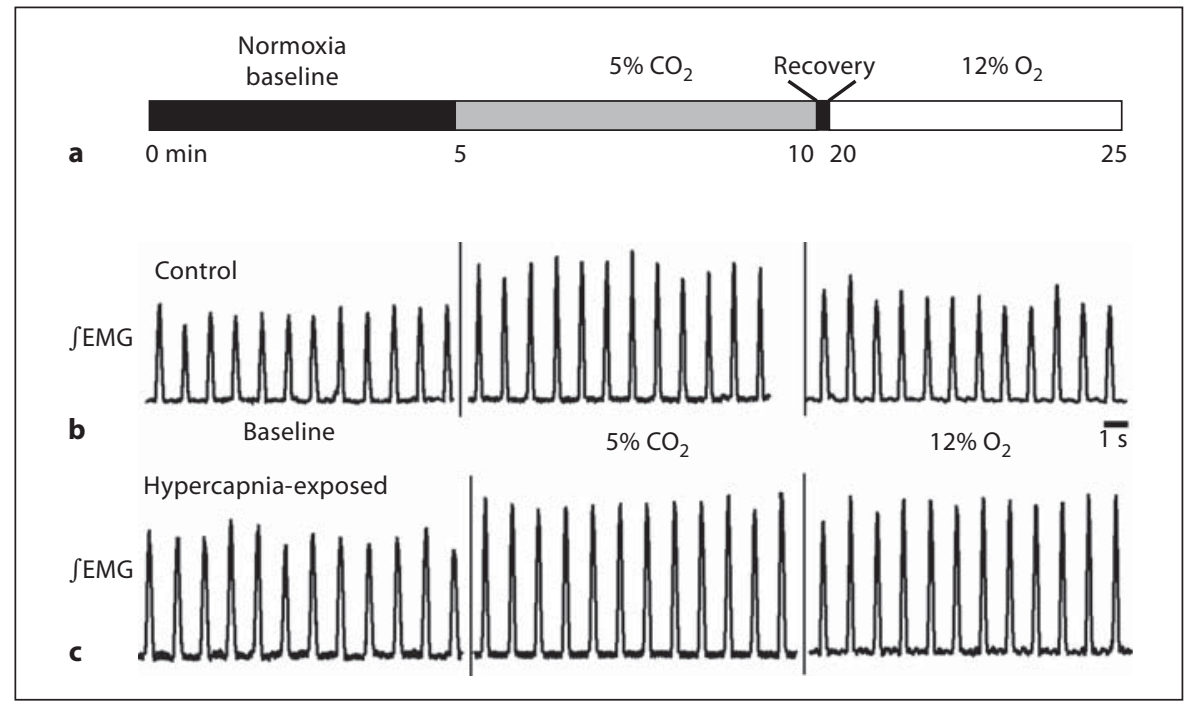

tion of each gas mixture. In response to acute hypercapnic and hypoxic exposures, data were analyzed at the peak response to the stimulus. This was determined by locating the maximal changes in amplitude and frequency of EMG measurements in response to each gas stimulus supplied. For all animals, this response occurred within 2 min of the onset of each gas exposure.

The minute EMG was calculated for both exposed and control animals during baseline, hypercapnia, and hypoxia. The calculation was done by first summing the area measurements of the peak breaths analyzed for integrated EMG and frequency. We then determined the length of each breath by calculating the number of data points in the integrated signal and dividing by the sampling frequency $(2,000$ samples/s). The number of breaths in $1 \mathrm{~min}$ was then determined by dividing this number by 60 . The sum of the areas was multiplied by this factor to determine the minute EMG. All data were normalized to the baseline values for each animal because of variability in the signal recorded between pups. Statistical significance was determined by using unpaired, single-tail $t$ tests when comparing data between groups. When comparing animals within the same group, statistical significance was determined with paired, single-tail $t$ tests. Multiple groups were compared using one-way ANOVA. All data are expressed as means \pm standard deviation and a $p$ value $<0.05$ was considered statistically significant. We used a relatively small number of animals in this study as was suggested with a power calculation based on strong effect in our early experiments.

\section{Results}

This study examined the effect of exposure to intermittent hypercapnia from postnatal days 7 to 14 versus normocapnic control conditions, on respiratory responses to a subsequent hypercapnic and hypoxic challenge in rat pups. Figure 1 provides a timeline for the previously described experimental protocol administered to each rat pup and representative integrated electromyographic signal recordings from control and hypercapnia-exposed animals.

In response to the hypercapnic challenge on day 15 , both groups of animals exhibited a significant increase in minute EMG from baseline measurements (fig. 2). The minute EMG during the hypercapnic response of control animals was $124 \pm 29 \%(\mathrm{p}=0.02)$; for exposed animals it was $120 \pm 26 \%(\mathrm{p}=0.01)$, when expressed as a percentage of baseline values, where the mean for baseline values is considered to be $100 \%$. There was no difference in responses of minute EMG between control and hypercapnia-exposed animals. We then evaluated both the integrated EMG and frequency components of minute EMGs. The integrated EMG increased from baseline for control animals $(131 \pm 30 \% ; \mathrm{p}=0.01)$ and exposed animals $(131$ $\pm 35 \% ; \mathrm{p}=0.005)$. Breathing frequency declined from baseline in both groups. The average breathing frequency of control animals decreased from a baseline value of 39.8 \pm 1.8 breaths per minute to $38.1 \pm 2.4$ breaths per minute $(\mathrm{p}=0.02)$. Hypercapnia-exposed animals had an average baseline frequency of $49.1 \pm 8.5$ breaths per minute, which decreased to $43.7 \pm 1.1$ breaths per minute in response to hypercapnia $(p=0.03)$. Integrated EMG and breathing frequency showed no significant differences between the groups in response to subsequent hypercapnic exposure.

We then examined the ventilatory responses of control and hypercapnia-exposed pups to a subsequent hypoxic challenge. The minute EMG for control animals 


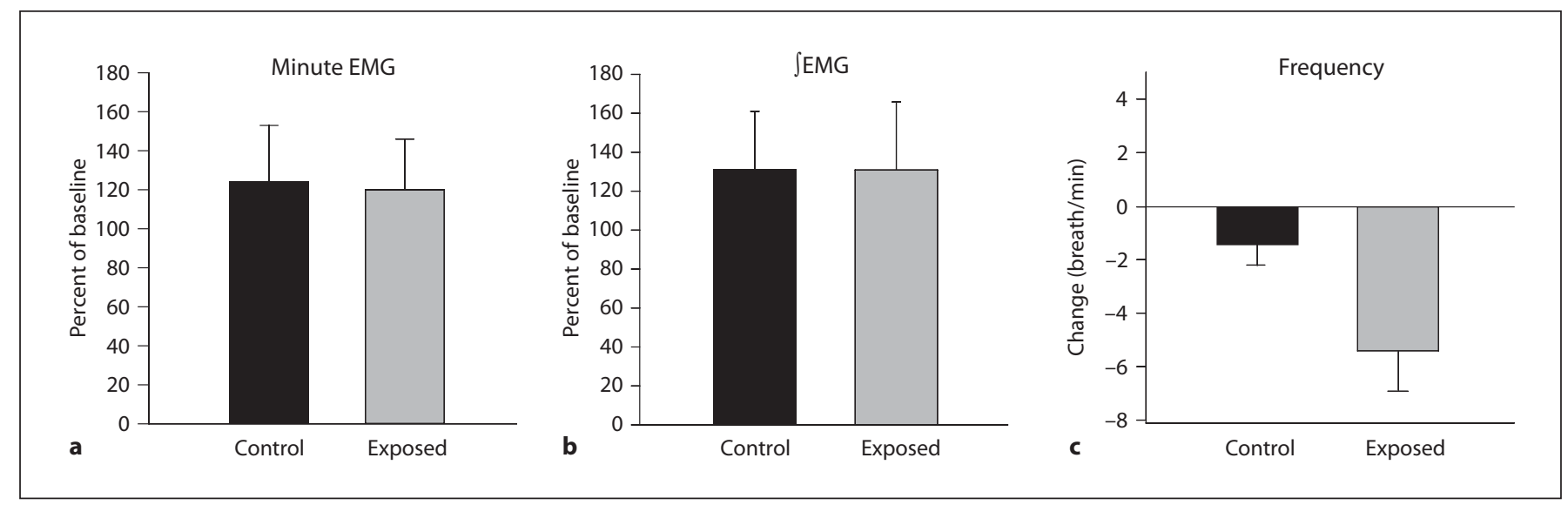

Fig. 2. Ventilatory responses to $5 \% \mathrm{CO}_{2}$ at postnatal day 15. a Normalized minute EMG of control and hypercapnia-exposed animals in response to hypercapnic challenge. b Normalized integrated EMG ( $\left.\int \mathrm{EMG}\right)$ of control and exposed animals during hypercapnic challenge. c Change in frequency from baseline rate during hypercapnic challenge. All values were significantly different from baseline values; however, there was no difference between control and exposed groups. Data are means \pm standard deviation.

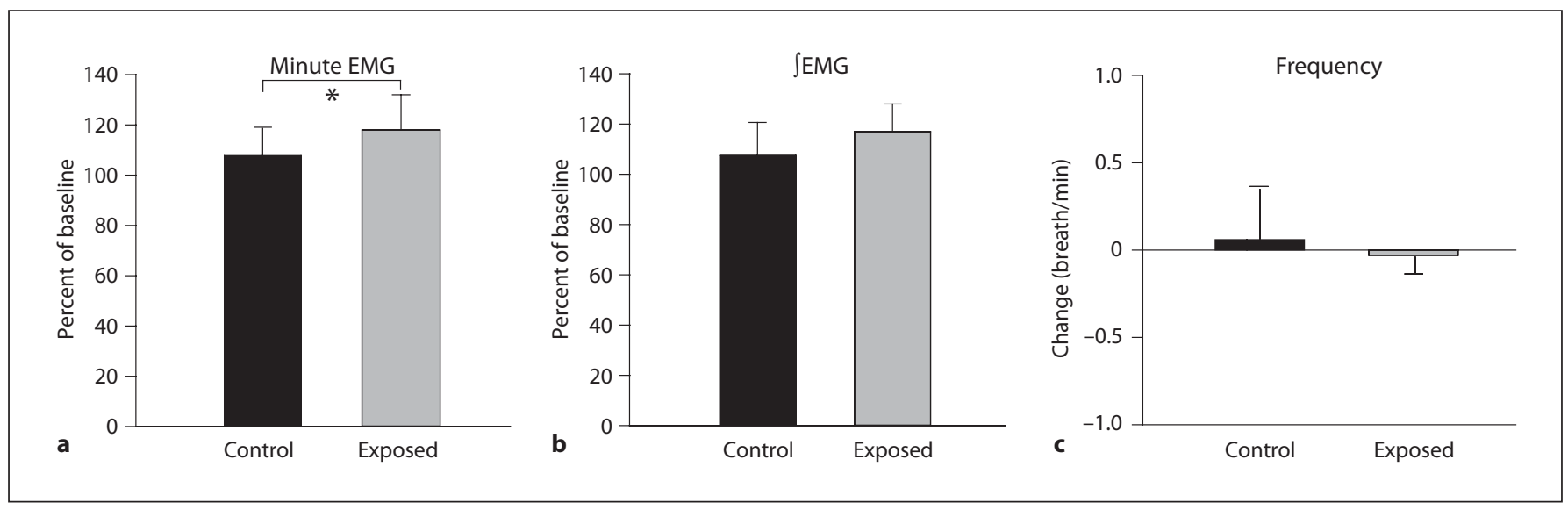

Fig. 3. Ventilatory responses to $12 \% \mathrm{O}_{2}$ at postnatal day 15. a Normalized minute EMG of control and hypercapnia-exposed animals in response to hypoxic challenge. The minute EMG response of hypercapnia-exposed animals was significantly higher than that of control animals $\left({ }^{*} \mathrm{p}<0.05\right)$. b Normalized integrated EMG $\left(\int E M G\right)$ of control and exposed animals during hypoxic challenge. c Change in frequency from baseline rate during hypoxic challenge. Data are means \pm standard deviation.

did not change from baseline in response to hypoxia (107 $\pm 13 \%$ ) (fig. 3). In contrast, hypercapnia-exposed animals showed an increase in minute EMG to $118 \pm 14 \%$ $(\mathrm{p}<0.001)$. The result was a significant difference in minute EMG response between control and exposed animals $(p<0.05)$. The integrated EMG response to hypoxia showed an increase from baseline in both control animals $(111 \pm 10 \% ; \mathrm{p}=0.01)$ and hypercapnia-exposed animals $(117 \pm 11 \%$; $p<0.001)$. The response of integrated EMG of hypercapnia-exposed pups showed no significant difference from the integrated EMG response of control animals. Neither control animals nor exposed animals showed a significant change in frequency from baseline values. 


\section{Discussion}

Preterm infants are typically exposed to episodes of hypercapnia and hypoxia due to immature respiratory control and ineffective ventilation. Previous research has focused on the ventilatory response to intermittent hypoxia and combined hypercapnia-hypoxia; however, the effects of intermittent hypercapnia alone have not been studied [15]. We therefore examined the effects of exposure to recurrent hypercapnia during early postnatal life, employing neonatal rat pups. We sought to test the hypothesis that exposure to intermittent hypercapnia during early postnatal life alters the response to subsequent hypercapnic and hypoxic exposures and may destabilize respiratory neural output. We found that rat pups exposed to recurrent hypercapnia exhibited enhanced ventilatory responses to a subsequent hypoxic challenge in comparison with controls while responses to a subsequent hypercapnic challenge were unaffected.

Immaturity of brain development at birth makes the rat pup a desirable animal model for preterm infants $[16$, 17]. We found that exposure to intermittent hypercapnia does not alter the ventilatory response to a subsequent hypercapnic challenge. In contrast, during the hypoxic challenge, the minute EMG response of intermittent-hypercapnia-exposed rat pups was significantly greater than that of control animals. The difference between the groups was primarily attributable to the increase in integrated EMG, which we are treating as synonymous with tidal volume since there was no difference in frequency. Our observation that the initial response of minute EMG to hypoxia is primarily due to an increase in amplitude rather than rate is consistent with the comparable hypoxic response in preterm infants [18]. Therefore, we speculate that our findings in rat pups are applicable to respiratory control in preterm infants.

Recurrent hypoxia has been extensively studied and has been shown to produce an increased ventilatory response to subsequent hypoxic exposures via tidal volume increases in rat pups [19]. Recent studies in preterm infants and rat pups have demonstrated that an increased frequency of apnea is associated with an increased ventilatory response to hypoxic challenges [11, 12, 20]. Increased peripheral chemoreceptor activity has been correlated with periodic breathing and disturbances of the respiratory control system in infants $[19,21]$. Rat pups show a similar destabilization of respiratory control with increased carotid body activity in response to chronic intermittent hypoxia $[10,22]$. Therefore, increased ventilatory response to hypoxia is associated with increased respiratory instability. Synergistic interactions between increased $\mathrm{CO}_{2}$ and lowered $\mathrm{O}_{2}$ likely contribute to destabilized breathing patterns [23].

In a previous study of piglets exposed to combined hypercapnic-hypoxic gas mixtures, ventilatory responses to subsequent hypoxic challenges were enhanced [13]. The elevated minute EMG response to subsequent hypoxia which we have observed in hypercapnia-exposed rat pups provides evidence that exposure to intermittent hypercapnia alone upregulates respiratory activity as is the case for intermittent hypoxic exposure. Nonetheless, these effects may be mediated by different pathways as hypercapnia increases ventilatory drive primarily through interaction with central chemosensors at the ventral brainstem while hypoxia alters the carotid body inputs to the respiratory pattern formation network $[10,22]$. We do not yet know what cellular mechanisms are responsible for ventilatory changes in response to intermittent hypercapnia, but a recent study suggests that a sodium-activated potassium channel may be directly involved in sensing hypercapnia and acidosis in brainstem neurons [24]. These channels seem to be logical targets for future evaluation of hypercapnia-induced changes in the brainstem respiratory neural network, particularly if their expression is selectively altered in brainstem areas responsible for integrating afferent input (nTS or even the pre-Bötzinger complex) and altering the breathing pattern. One obvious progression of this work would be to record from respiratory related neurons within the nTS or ventral respiratory group in intermittent hypercapnia-exposed animals and evaluate the participation of sodium-activated potassium channels in neuronal activity. Regardless of cellular mechanism, we speculate that altered chemosensation in response to both intermittent hypercapnia and hypoxia may contribute to instability of breathing in neonates subject to recurrent apnea. Further studies might address whether this effect of intermittent hypercapnia is long lasting as former preterm infants are at risk of developing sleep-disordered breathing in childhood, by as yet unclear mechanisms [25, 26].

Several limitations of this study need to be considered. For this study, we chose to study rat pups that were in their second week because apneic episodes in preterm infants typically develop beyond the first days of life, although this gradual increase in apnea frequency may be related to resolution of respiratory distress syndrome over the first days of life [27]. Additionally, steady-state increases in ventilation after hypercapnic exposure have been shown to extend beyond $2 \mathrm{~min}$ [28] in human infants. While this phenomenon did not appear to occur in 
our data, our observed ventilatory response to hypercapnia, consisting of an increase in amplitude and decrease in frequency, was still consistent with what has been previously documented in infants $[7,8]$. During the intervention period for hypercapnia-exposed rat pups, the duration of the intermittent hypercapnic exposures and cycle times were arbitrarily determined. We must further acknowledge that unrecognized previous hypercapnic and/or hypoxic exposures may have had an effect of unknown magnitude since we did not measure changes in minute ventilation or frequency during the intermittent hypercapnia exposure. Additionally, we were unable to measure blood gas levels prior to termination of the experimental protocol and therefore cannot determine the extent of hypercapnia the pups experienced during intermittent hypercapnia or acute hypercapnia and hypoxia challenges. We also considered the potential effects of the anesthetic on respiratory control; however, both control and hypercapnia-exposed animals underwent the same surgical preparation and acute hypercapnic and hypoxic exposures, thereby negating the notion that any advantages were gained by either group of rat pups and providing a similar background for the anesthetic 'state' of the animal. Procedures were well tolerated by the pups and only a modest number of pups with potentially compromised blood gas status at the completion of the protocol were discarded prior to any data analysis.

In summary, we have shown that neonatal exposure to intermittent hypercapnia alters the ventilatory response to subsequent hypoxic challenge while the response to hypercapnia is unchanged. As enhanced ventilatory responses to hypoxia have been associated with unstable breathing patterns, our physiologic findings suggest that intermittent hypercapnia also contributes to destabilizing respiratory control during early postnatal life. We look to future studies to uncover cellular and neural mechanisms that play a role in the altered ventilatory response presented in this study, as well as to examine the long-term effects of neonatal exposure to intermittent hypercapnia on respiratory control mechanisms.

\section{Acknowledgement}

This study was funded by the NIH-National Heart, Lung, and Blood Institute grant: HL081622 awarded to C.G.W.

\section{References}

1 Martin RJ, Abu-Shaweesh JM: Control of breathing and neonatal apnea. Biol Neonate 2005;87:288-295.

$\checkmark 2$ Gerhardt T, Bancalari E: Apnea of prematurity. I. Lung function and regulation of breathing. Pediatrics 1984;74:58-62.

-3 Durand M, Cabal LA, Gonzalez F, Georgie S, Barberis C, Hoppenbrouwers T, Hodgman J: Ventilatory control and carbon dioxide response in premature infants with idiopathic apnea. Am J Dis Child 1985;139:717-720.

4 Abu-Shaweesh JM, Dreshaj IA, Thomas AJ, Haxhiu MA, Strohl KP, Martin RJ: Changes in respiratory timing and induced by hypercapnia in maturing rats. J Appl Physiol 1999; 87:484-490.

$\checkmark 5$ Krauss AN, Klain DB, Waldman S, Auld P: Ventilatory response to carbon dioxide in newborn infants. Pediatr Res 1975;9:46-50.

-6 Rigatto H, Brady JP, Verduzco R: Chemoreceptor reflexes in preterm infants. II. The effects of gestational and postnatal age on the ventilatory response to inhaled carbon dioxide. Pediatrics 1975;55:614-620.

-7 Martin RJ, Carlo WA, Robertson SS, Day WR, Bruce EN: Biphasic response of respiratory frequency to hypercapnia in preterm infants. Pediatr Res 1985;19:791-796.
8 Noble LM, Carlo WA, Miller MJ, DiFiore JM, Martin RJ: Transient changes in expiratory time during hypercapnia in premature infants. J Appl Physiol 1987;62:1010-1013.

$\checkmark 9$ Coates EL, Silvis ML: Age-related changes in the ventilatory response to inspired $\mathrm{CO}_{2}$ in neonatal rats. Respir Physiol 1999;118:173179.

10 Peng YJ, Rennison J, Prabhakar NR: Intermittent hypoxia augments carotid body and ventilatory response to hypoxia in neonatal rat pups. J Appl Physiol 2004;97:2020-2025.

11 Nock ML, DiFiore JM, Arko MK, Martin RJ: Relationship of the ventilatory response to hypoxia with neonatal apnea in preterm infants. J Pediatr 2004;144:291-295.

12 Cardot V, Chardon K, Tourneux P, Micallef S, Erwan S, Leke A, Bach V, Libert JP, Telliez $\mathrm{F}$ : Ventilatory response to a hyperoxic test is related to the frequency of short apneic episodes in late preterm neonates. Pediatr Res 2007;62:591-596.

$\checkmark 13$ Waters KA, Tinworth KD: Depression of ventilatory responses after daily, cyclic hypercapnic hypoxia in piglets. J Appl Physiol 2001;90:1065-1073.
14 Mayer CA, Haxhiu MA, Martin RJ, Wilson CG: Adenosine $A_{2 A}$ receptors mediate GABAergic inhibition of respiration in immature rats. J Appl Physiol 2006;100:91-97.

15 Bavis RW: Developmental plasticity of the hypoxic ventilatory response after perinatal hyperoxia and hypoxia. Respir Physiol Neurobiol 2005;149:287-299.

16 Dobbing J: Later development of the brain and its vulnerability; in Davis JA, Dobbing J (eds): Scientific Foundations of Pediatrics. Philadelphia, Saunders, 1974, pp 567-568.

17 Matsuoka T, Mortola JP: Effects of hypoxia and hypercapnia on the Hering-Breuer reflex of the conscious newborn rat. J Appl Physiol 1995;78:5-11.

18 Martin RJ, DiFiore JM, Jana L, Davis RL, Miller MJ, Coles SK, Dick TE: Persistence of the biphasic ventilatory response to hypoxia in preterm infants. J Pediatr 1998;132:960964.

19 Bavis RW, Olson EB Jr, Vidruk EH, Fuller DD, Mitchell GS: Developmental plasticity of the hypoxic ventilatory response in rats induced by neonatal hypoxia. J Physiol 2004; 557:645-660. 
20 Julien C, Bairam A, Joseph V: Chronic intermittent hypoxia reduces ventilatory longterm facilitation and enhances apnea frequency in newborn rats. Am J Physiol Regul Integr Comp Physiol 2008;294:R1356R1366.

-21 Al-Matary A, Kutbi I, Qurashi M, Khalil M, Alvaro R, Kwiatkowski K, Cates D, Rigatto $\mathrm{H}$ : Increased peripheral chemoreceptor activity may be critical in destabilizing breathing in neonates. Semin Perinatol 2004;28: 264-272.
-22 Pawar A, Peng YJ, Jacono FJ, Prabhakar NR: Comparative analysis of neonatal and adult rat carotid body responses to chronic intermittent hypoxia. J Appl Physiol 2008;104: 1287-1294.

23 Khan A, Qurashi M, Kwiatkowski K, Cates $\mathrm{D}$, Rigatto $\mathrm{H}$ : Measurement of the $\mathrm{CO}_{2}$ apneic threshold in newborn infants: possible relevance for periodic breathing and apnea. J Appl Physiol 2005;98:1171-1176.

24 Ruffin VA, Gu XQ, Zhou D, Douglas RM, Sun X, Trouth CO, Haddad GG: The sodium-activated potassium channel Slack is modulated by hypercapnia and acidosis Neuroscience 2008;151:410-418.

$\checkmark 25$ Rosen CL, Larkin EK, Kirchner HL, Emancipator JL, Bivins SF, Surovec SA, Martin MJ, Redline S: Prevalence and risk factors for sleep-disordered breathing in 8- to 11-yearold children: association with race and prematurity. J Pediatr 2003;142:383-389.
26 Reeves SR, Gozal D: Developmental plasticity of respiratory control following intermittent hypoxia. Respir Physiol Neurobiol 2005; 149:301-311.

$>27$ Carlo WA, Martin RJ, Versteeg FGA, Robertson SS, Goldman MD, Fanaroff A: The effect of respiratory distress syndrome on chest wall movements and respiratory pauses in preterm infants. Am Rev Respir Dis 1982; 126:103-107.

28 Anderson JV, Martin RJ, Abboud EL, Dyme IZ, Bruce EN: Transient ventilatory response to $\mathrm{CO}_{2}$ as a function of sleep state in term infants. J Appl Physiol Respir Environ Exercise Physiol 1983:54:1482-1488. 